\title{
Canonical Wnt signaling activity during synovial joint development
}

\author{
Takashi Yamagami · Andrei Molotkov • \\ Chengji J. Zhou
}

Received: 6 October 2009/Accepted: 9 November 2009/Published online: 18 November 2009

(C) The Author(s) 2009. This article is published with open access at Springerlink.com

\begin{abstract}
Wnt signaling plays important roles in skeletal development. However, the activation and function of canonical Wnt signaling in joint development remains unclear. We analyzed the lineage identity and developmental changes of the Wnt-responsive cells during synovial joint formation as well as adulthood in the Wnt signaling reporter TOPgal transgenic mice. At embryonic day (E) 12.5, we found that the TOPgal was inactivated in the presumptive joint forming interzone, but it was intensively activated in the cartilage anlage of developing long bones and digits. At E14.5, the TOPgal activity was found in a subgroup of the articular chondrocyte lineage cells, which were co-immunolabeled with Doublecortin intensively and with Vinculin weakly. At E18.5, the TOPgal/ Doublecortin co-immunolabeled cells were found in the superficial layer of the developing articular cartilage. During postnatal development, the TOPgal(+) articular chondrocytes were abundant at P7 and decreased from P10. A small number of TOPgal(+) articular chondrocytes were also found in adult joints. Our study suggests an age- and
\end{abstract}

Takashi Yamagami and Andrei Molotkov contributed equally to this paper.

T. Yamagami · C. J. Zhou

Department of Cell Biology and Human Anatomy, School

of Medicine, University of California, Davis, CA, USA

A. Molotkov · C. J. Zhou ( $₫)$

Institute for Pediatric Regenerative Medicine, Shriners Hospitals for Children-Northern California, Room 602B, 2425 Stockton

Blvd., Sacramento, CA 95817, USA

e-mail: cjzhou@ucdavis.edu lineage-specific role of canonical Wnt signaling in joint development and maintenance.

Keywords Articular condrocytes - Synovial joints $\mathrm{Wnt} / \beta$-catenin reporter TOPgal transgenic mice . Doublecortin · Vinculin · Wnt4

\section{Introduction}

Genetic studies have demonstrated that Wnt signaling plays crucial roles in bone development (Baron et al. 2006; Krishnan et al. 2006; Day and Yang 2008; Leucht et al. 2008; Liu et al. 2008). Limb skeletal elements develop from cartilage anlage through endochondral bone formation (Karsenty and Wagner 2002; Kronenberg 2003), and both canonical and noncanonical Wnt signaling pathways have been implicated in this process (Church and Francis-West 2002; Yang 2003; Hartmann 2006). Synovial joints are the most common and most movable joints connecting two or more bones for skeletal function. Its development starts with the formation of the interzone at the future joint site, which divides the mesenchymal condensation (Archer et al. 2003; Pitsillides and Ashhurst 2008). The cells within the interzone differentiate into various tissues of the synovial joint, including the articular cartilage, ligaments, synovium, and fibrous capsules. The neighboring cells in the mesenchymal condensation differentiate into the growth plate chondrocytes. Although much is currently known about long bone development, the mechanisms underlying joint formation remains little understood. Several Wnts (including Wnt4, Wnt9a, and Wnt16) are expressed in the interzone and are required for joint formation (Guo et al. 2004; Spater et al. 2006a, b). However, the Wnt-responsive cells during joint development remains to be characterized. 
The canonical Wnt signaling pathway regulates the ability of $\beta$-catenin to activate the transcription of $\mathrm{Wnt}$ target genes (Gordon and Nusse 2006). In the absence of Wnt signaling, the cytoplasmic $\beta$-catenin is phosphorylated by Gsk $3 \beta$ in an intracellular complex and degraded by ubiquitination. When Wnts bind to the receptor Fzds and Lrp5/6, the intracellular $\beta$-catenin is stabilized and translocated into the nucleus where it binds to the transcriptional Tcf/Lef complex. The Wnt signaling reporter TOPgal mice, which express the lacZ gene under the control of multiple Tcf/Lef binding sites (the Wnt-responsive elements) (DasGupta and Fuchs 1999), have been widely and reliably used to determine the activation site of the canonical Wnt/ $\beta$-catenin signaling pathway (Barolo 2006). Combined with lineage-specific markers, we analyzed the lineage identity and developmental changes of the Wntresponsive cells during synovial joint formation as well as adulthood in the TOPgal mice.

\section{Materials and methods}

\section{Animals}

The Wnt reporter TOPgal mice were generated by Fuchs lab (DasGupta and Fuchs 1999) and distributed by the Jackson Laboratory (Bar Harbor, Maine, USA) (Stock number 004623, Tg(Fos-lacZ)34Efu/J). The mice were housed in the vivarium of the UC Davis Medical School (Sacramento, CA). Timed-mated, pregnant TOPgal female mice were euthanized with $\mathrm{CO}_{2}$ gas prior to cesarean section. The embryos were immersion-fixed in $4 \%$ paraformaldehyde (PFA) solution at $4^{\circ} \mathrm{C}$ overnight. The day of conception was designated embryonic day 0 (E0). Postnatal mouse pups and adults were deeply anesthetized with isoflurane and transcardially perfused with $4 \%$ PFA. The day of birth was designated postnatal day 0 (P0). All research procedures using mice were approved by the UC Davis Animal Care and Use Committee and conformed to NIH guidelines.

\section{Detection of TOPgal activity by X-gal staining}

The dissected limbs were immersed into X-gal (5-bromo-4chloro-3-indolyl-beta-D-galactopyranoside) substrate solution (with $1 \mathrm{mg} / \mathrm{ml} \mathrm{X}$-gal in $5 \mathrm{mM} \mathrm{K} \mathrm{K}_{4} \mathrm{Fe}(\mathrm{CN})_{6}, 5 \mathrm{mM}$ $\mathrm{K}_{3} \mathrm{Fe}(\mathrm{CN})_{6}$, and $5 \mathrm{mM} \mathrm{MgCl}_{2}$ ) for $12-24 \mathrm{~h}$ to detect the enzymatic activity of $\beta$-galactosidase. The stained embryonic limbs were embedded in $3 \%$ agarose and sectioned at $100 \mu \mathrm{m}$ with a vibratome (Leica VT1000S, Leica Microsystems, Germany). The stained postnatal joints were embedded in paraffin wax, sectioned $10 \mu \mathrm{m}$ with a microtome, and counterstained with hematoxylin and eosin (for P10) or eosin only (for adults).

Immunohistochemistry and in situ hybridization

The fixed samples were cryoprotected in 30\% sucrose, embedded in OCT compound (Tissue-Tek, Sakura, Japan), frozen in a mixture of $100 \%$ ethanol and dry ice, and sectioned on a Leica cryostat at $12 \mu \mathrm{m}$. Sections were mounted onto SuperFrost microscope slides (Fisher Scientific, USA) and stored at $-80^{\circ} \mathrm{C}$ freezers until used. Samples from E18.5 embryos and postnatal animals were decalcified for 2-3 days in the Immunocal solution (Decal Chemical Corp., Cogers, NY, USA) before embedding.

The immunohistochemistry was carried out according to the standard procedures as previously described (Wang et al. 2008). Briefly, the tissue sections were preincubated with a blocking solution of $10 \%$ lamb serum in phosphate-buffered saline (PBS) for $2 \mathrm{~h}$ at room temperature. The sections were incubated with one or two of the following antibodies: (1) the rabbit antiserum for $\beta$-galactosidase (1:1000, Cappel, MP Biomedicals, Solon, $\mathrm{OH}$, USA), (2) the mouse antiserum for Vinculin (1:100, Santa Cruze Biotechnology, CA, USA), and (3) the guinea pig antiserum for Doublecortin (DCX) (1:100, Abcam, Cambridge, MA, USA). The primary antibodies were diluted in $1 \%$ lamb serum in PBS. After overnight incubation at $4^{\circ} \mathrm{C}$ with primary antibodies, sections were rinsed in PBS three times for $10 \mathrm{~min}$ each and incubated for $2 \mathrm{~h}$ at room temperature with the secondary antibodies. The following secondary antibodies were used: (1) Alexa Fluor 488 goat anti-rabbit IgG, (2) Alexa Fluor 594 goat anti-guinea pig IgG, (3) Alexa Fluor 594 goat anti-mouse IgG (Molecular Probes, Inc., Eugene, OR, USA; all diluted in 1:1000). After three 10-min washes with PBS, sections were counterstained with DAPI $(1 \mathrm{mg} / \mathrm{ml})$ and mounted with Vectashield (Vector Laboratories, Burlingame, CA, USA). In situ hybridization was performed with digoxigenin labeled RNA probes as described previously (Wang et al. 2008).

Fluorescence and confocal microscopy

All tissue sections were assessed using a Zeiss Axiophot 2 fluorescence microscope equipped with AxioCam digital camera (Carl Zeiss, Inc., North America). Confocal images were obtained using a Nikon Eclipse C1 confocal microscope system (Nikon, North America). Images were processed using Adobe Photoshop (version CS2 for Macintosh) to equalize image brightness and contrast. Image composition was not altered in any way. 


\section{Results and discussion}

The Wnt reporter TOPgal activity was found intensively in long bone anlage but absent in the Wnt4-expressing presumptive joint interzone during early embryonic development

The presumptive joint interzone is distinguishable around E12.5 in the mouse forelimbs with down-regulated expression of a chondrocyte marker Col2al and the clustered high expression of the joint markers Gdf5 and Wnt4 in the future joint region (Spater et al. 2006a). At this early age, X-gal staining for TOPgal activity was detected intensively in the chondrogenic condensation of the scapula, humerus, ulna, and radius (Fig. 1a, b). However, the $\mathrm{X}$-gal staining was not found in areas where the shoulder and elbow joints were forming, which was evident by Wnt4 expression (Fig. 1c, d). At E14.5, the pattern of X-gal staining was similar to what observed at E12.5. Intensive $\mathrm{X}$-gal staining was observed in the developing long bones and digits; whereas, no or only weak X-gal staining was present in the developing joints (Fig. 1e-g). Absence of
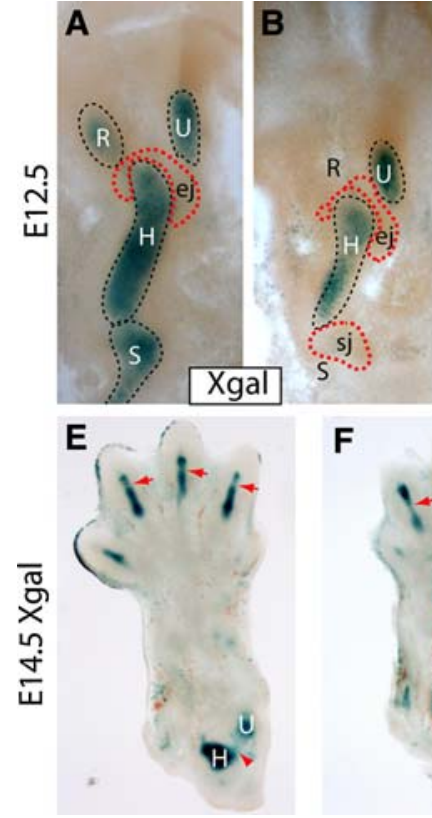

$\mathbf{F}$
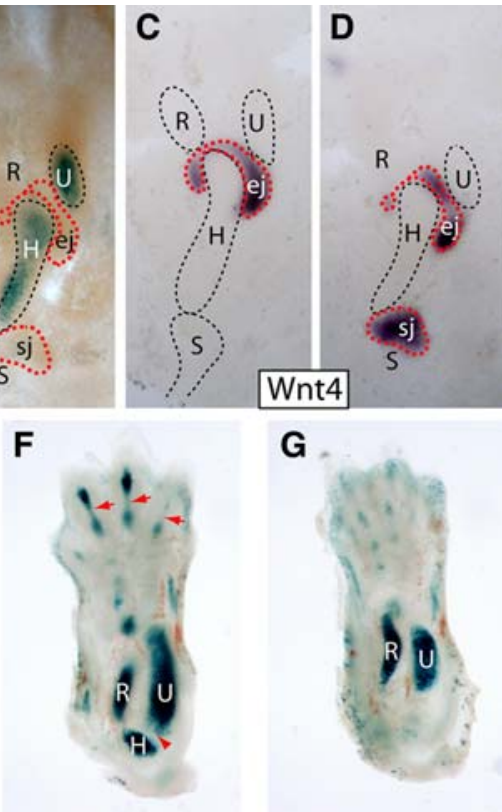

G

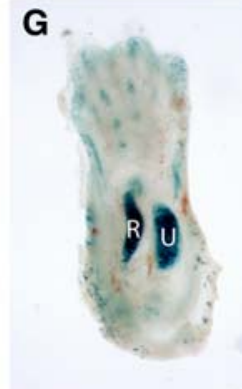

Fig. 1 X-gal staining of the Wnt reporter TOPgal and in situ hybridization of Wnt 4 during early joint development. a, b X-gal staining of two representative forelimb sections of the E12.5 TOPgal embryo showed the canonical Wnt signaling activity in the cartilage anlage of developing long bones and digits but not in the presumptive joint forming regions. c, d In situ hybridization signals for the noncanonical Wnt4 were detected only in the presumptive joint forming regions of the E12.5 forelimb sections. e-g X-gal staining of three serial sections of the E14.5 TOPgal forelimbs showed the signals in the presumptive forming elbow joint (arrowheads) and interdigital joints (arrows). ej presumptive elbow joint, $H$ humerus, $R$ radius, $S$ scapula, sj presumptive shoulder joint, $U$ ulna
X-gal staining in regions where synovial joints were forming suggests that a high activity of canonical Wnt signaling is not required for the induction or maintenance of the interzone at this early developmental stage.

Wnt9a (formerly named as Wnt14) was expressed in the early joint interzone in both chick and mouse embryos, and gain-of-function studies in these organisms suggest a role of Wnt9a in the induction of joint interzone probably upstream of the joint forming gene Gdf5 (Hartmann and Tabin 2001; Guo et al. 2004). However, the loss-of-function studies demonstrated that Wnt9a, Wnt 4 , or $\beta$-catenin were not required for joint induction but likely for the subsequent maintenance of the cell fate in the joint interzone (Spater et al. 2006a, b). Genetic deletion of Wnt9a in mice did not affect the expression of joint marker genes, but it resulted in synovial chondroid metaplasia in some joints; and the joint phenotype was enhanced in the double knockout embryos of Wnt9a and Wnt4 (Spater et al. 2006a, b). Conditional deletion of $\beta$-catenin by Col2a1-Cre in mesenchymal/chondrocyte lineage cells did not diminish but upregulated the expression domain of the early joint marker genes including Gdf5, Wnt4, and Gli1 (Spater et al. 2006a). Together, these data suggest that Wnt 4 and Wnt9a act cooperatively during joint development to control the fate of the joint interzone cells, possibly acting through the canonical $\mathrm{Wnt} / \beta$-catenin signaling pathway. However, the current study demonstrates that the canonical $\mathrm{Wnt} / \beta$ catenin signaling pathway is inactive in the joint interzone cells. Indeed, Wnt4 is classified as a noncanoical Wnt and has been demonstrated to inhibit $\beta$-catenin/Tcf signaling (Veeman et al. 2003; Bernard et al. 2008), which suggests a possible mechanism of the inactivation of $\mathrm{Wnt} / \beta$-catenin signaling pathway in the joint interzone during early embryonic development.

The Wnt reporter TOPgal was activated in articular chodrocyte lineage cells which displayed dynamic changes of positional and antigenic characters during late gestation

Articular chondrocytes play crucial roles in the development and maintenance of synovial joints. DCX was recently demonstrated to be specifically expressed in the articular chondrocyte lineage cells but not in the growth plate chondrocytes (Zhang et al. 2007). Therefore, we used DCX antibodies to characterize the TOPgal-activated cells in the embryonic articular cartilage. In both elbow and knee joints at E14.5, we found that the TOPgal $(+) /$ $\mathrm{DCX}(+)$ cells were located in the intermediate layer of the developing articular cartilage (Fig. 2a-c), while more DCX $(+) /$ TOPgal $(-)$ cells were located in the outer layer. These results suggest that canonical Wnt signaling pathway is activated in a restricted subpopulation of $\mathrm{DCX}(+)$ 
Fig. 2 Double fluorescence immunolabeling of TOPgal activity with an articular condrocyte marker DCX and a mesenchymal marker Vinculin at E14.5. a-c $\beta \mathrm{gal}+/ \mathrm{DCX}+$ co-immunolabeled articular condrocytes were found in the intermediate layer, while the $\beta$ gal-/DCX + articular condrocytes were located in the outer layer of the elbow joint.

* $\beta$ gal+/DCX- condrocyte precursors. d-j Double immunolabeling of $\beta \mathrm{gal}$ and Vinculin on the sections of elbow joint $(\mathbf{d}-\mathbf{g})$ and knee joint $(\mathbf{h}-\mathbf{j})$ at E14.5. Panels $\mathbf{e}-\mathbf{g}$ were enlarged from the dashed rectangle in d. Intense $\beta \mathrm{gal}$ positive articular condrocytes were co-immunolabeled with weak Vinculin in the intermediate layer (indicated by asterisks and arrowheads within two dashed curves)
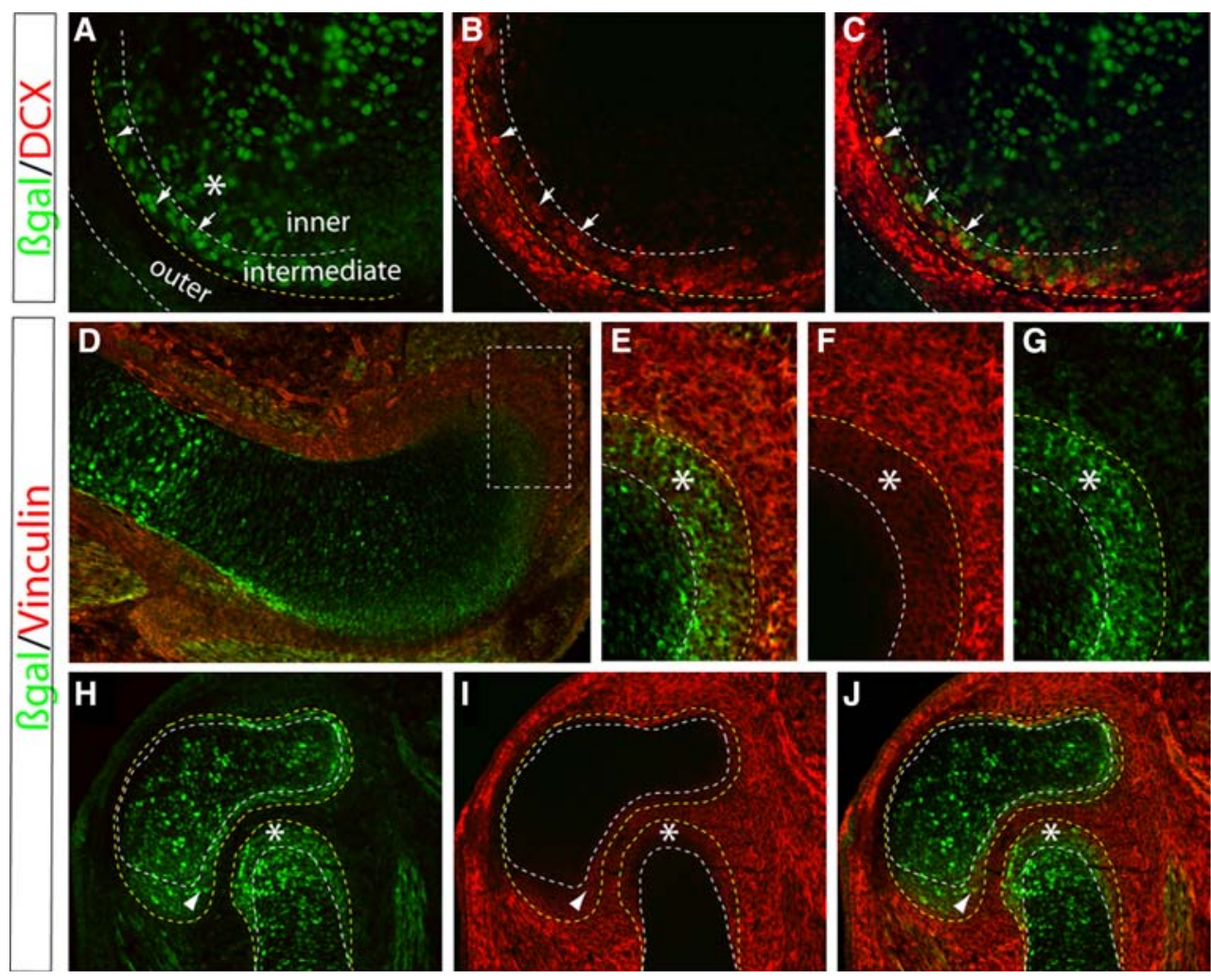

articular chondrocytes in the forming articular cartilage during mid-gestation. Notably, numerous TOPgal $(+) /$ DCX $(-)$ cells were found in the inner layer of the forming articular cartilage at this developmental stage (Fig. 2a-c). The TOPgal $(+)$ articular chondrocytes were also weakly co-immunolabeled with a mesenchymal marker Vinculin (Fig. $2 \mathrm{~d}-\mathrm{j}$ ), which the later showed intensive immunolabeling in the mesenchymal cells outside of the developing bones and no immunolabeling in both inner cartilarge and growth plate chondrocytes. These results suggest that the Wnt-responsive articular chondrocytes were originated from the Vinculin(+) mesenchymal cells.

At E18.5, the TOPgal(-)/DCX(+) outer layer no longer existed in the forming articular cartilage in the elbow joints; Instead, the TOPgal $(+) / \mathrm{DCX}(+)$ articular chondrocytes presented in the superficial layer of the developing epithysis (Fig. 3a-d). At the same age, the TOPgal $(+) /$ Vinculin(+) cells were only found in a single-cell layer of the perichondrium restricted to the peripheral region of the forming joints (Fig. 3e-h).

The dynamic changes of the positional and antigenic characters of the TOPgal $(+)$ cells in the articular cartilage of the limb joints from E14.5 to E18.5 may reflect the differentiation process of the articular chondrocytes. That is, the Vinculin $(+)$ joint interzone mesenchymal cells differentiate into $\mathrm{DCX}(+)$ articular chodrocyte precursors, which subsequently differentiate into $\mathrm{DCX}(+) / \mathrm{TOPgal}(+)$ progenitors and $\mathrm{DCX}(-) / \mathrm{TOPgal}(+)$ immature articular chodrocytes, from the surface to the inner layer of the developing articular cartilage.

This suggestive conclusion is partially supported by a recent cell fate study that used Gdf5-Cre mice mated with ROSA-LacZ mice (Koyama et al. 2008). The growth differentiation factor Gdf5 is restrictively expressed in the interzone cells at the initial stage of the joint formation (Merino et al. 1999; Storm and Kingsley 1999). Indeed, the Gdf5-Cre/ROSA-LacZ reporter-expressing cells were originally found in the joint interzone cells and subsequently predominant in articulating layers and accessory joint tissues (Koyama et al. 2008). The same study also used both Wnt reporters BATgal and TOPgal to show the Wnt responsive cells in some embryonic joints, but they reported neither the inactivation of the Wnt reporters in the joint interzone cells during early gestation nor the antigenic characterizations and their dynamic changes. They also carried out the loss-of-function study by conditional deletion of $\beta$-catenin with either Gdf5-Cre or Col2a1-Cre, which demonstrated the formation of synovial joints in the mutants with a defective surface layer in the embryonic articular cartilage (Koyama et al. 2008). Taken together, these data suggest that canonical Wnt signaling is not required for the initial joint specification and formation but it is required for the articular chondrocyte development during late gestation. However, it remains unknown how Wnt signaling regulates this process. $\beta$-catenin is also essential in cell adhesion. Further study using our recently 

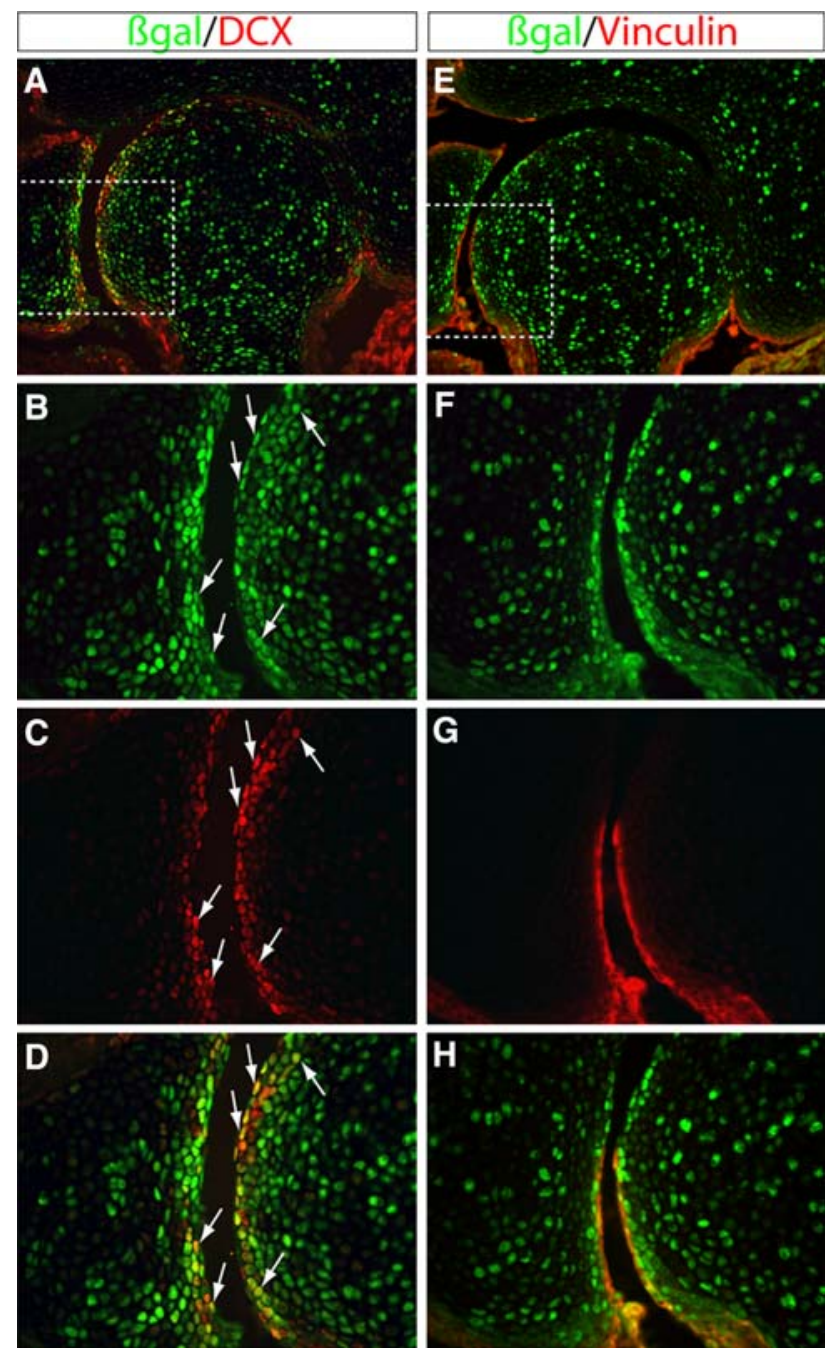

Fig. 3 Double fluorescence immunolabeling of TOPgal activity with DCX and Vinculin in the elbow joint at E18.5. a-d $\beta$ gal+/DCX+ co-immunolabeled articular condrocytes (arrows) were found in the surface and adjacent inner layer of the elbow joint. Panels b-d were enlarged from the dashed rectangle in a. e-h Some $\beta$ gal+/Vinculin + cells were restricted to the surface of proximal edge of the elbow joint at E18.5. Panels $\mathbf{f}-\mathbf{h}$ were enlarged from the dashed rectangle in $\mathbf{e}$

generated conditional Lrp6 mice (Zhou et al. 2009) may uncover the role of canonical Wnt signaling in the articular chondrocyte lineage cells during synovial development.

The Wnt signaling reporter TOPgal(+) articular chondrocytes reached a peak during the first postnatal week and existed a small population in adult limb joints

We performed X-gal staining to investigate the TOPgal(+) articular chondrocytes during postnatal joint development and in the adult articular cartilage. In the first postnatal week, we found a predominant expansion of the TOPgal $(+)$ articular chondrocytes in the surface layer of the articular cartilage as evident in the femur epithysis of the knee joints at P3 and P7 (Fig. 4a-e). Interestingly, some TOPgal $(+)$ cells were found in the trochlear groove transiently at P3 but absent at P7 (Fig. 4c, e). At P10, the TOPgal (+) articular chondrocytes were obviously reduced in the femur epiphysis and rarely found in the tibia epithysis in the knee joints (Fig. 4f, g). In the adult knee joint, we found a small group of TOPgal $(+)$ cells in the articular cartilage of the femur (Fig. 4h, i).

The large population of the TOPgal $(+)$ cartilage chondrocytes during early postnatal development suggests an age-specific role of canonical Wnt signaling probably for a rapid expansion of these cells to build up the functional limb joints within the first postnatal week of the mice. The
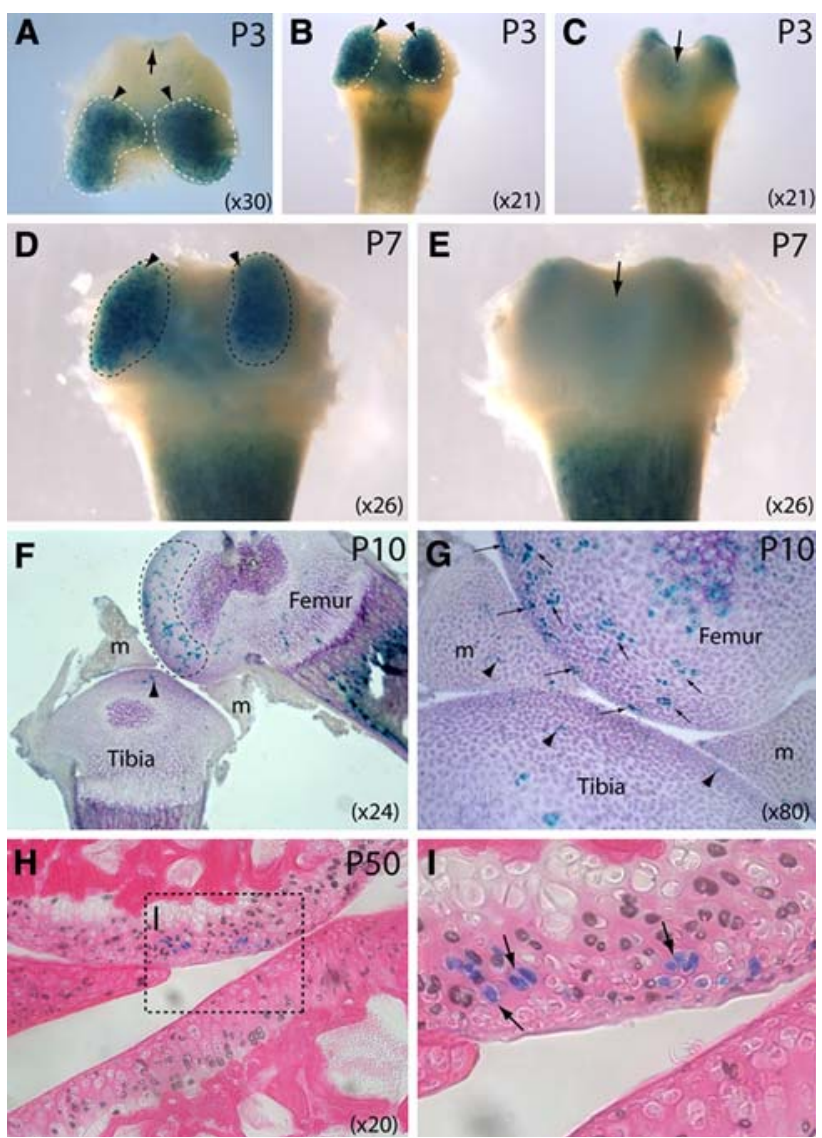

Fig. 4 X-gal staining for TOPgal activity in postnatal knee joints. a-e Abundant X-gal stained signals (arrowheads) were found in the transparent articular cartilage of the P3 (a, b) and P7 (d) femoral condyle (dash line area) of the TOPgal mouse femur; some X-gal stained signals were also found in the trochlear groove at P3 (arrows in $\mathbf{a}, \mathbf{c}$ ) but absent at P7 (arrow in e). f, g Many X-gal stained condrocytes (dash line area in $\mathbf{f}$ and arrows in $\mathbf{g}$ ) were scattered in the articular cartilage of the P10 femoral condyle; only a few X-gal stained condrocytes (arrowheads) were found in the articular cartilage of the tibial plateau and the meniscus (m). Sections were counterstained by HE staining. $\mathbf{h}$, i A small number of X-gal stained cells were found in the adult cartilage of the knee joint (arrows in $\mathbf{i}$ which is enlarged from the dashed square in $\mathbf{h}$ ). Sections were counterstained by eosin staining 
small population of TOPgal( + ) cells in the adult articular cartilage suggests that Wnt signaling may maintain an adult stem cell/progenitor pool for the articular cartilage repair and regeneration. Indeed, Wnt signaling has been implicated in several tissues/organs to maintain the adult stem cell niches, particularly the skin and intestinal stem cells (Reya and Clevers 2005; Fuchs 2008). Further studies may reveal a common role of the Wnt signaling in adult stem cell niches in various tissues/organs including the articular cartilage.

In conclusion, the current study demonstrated that the canonical Wnt signaling reporter TOPgal is not activated in the Wnt4-expressing joint interzone at E12.5, but it is activated in the articular chondrocyte lineage cells with dynamic changes of positional and antigenic characters from E14.5 to E18.5. We further demonstrated that the TOPgal(+) articular chondrocytes are increased predominantly within the first postnatal week and then reduced from P10. In addition, we found a small population of the TOPgal(+) chondrocytes in the adult joint cartilage. The activation site and developmental changes of the TOPgal activity may reflect the lineage- and age-specific roles of canonical Wnt signaling in synovial joint development and homeostasis.

Acknowledgments The authors thank Denys Rybka, Naficeh Dastgheyb, Kai Wang, and Jason Chan for technical assistance, Elsie Lodde for critical reading of this manuscript, and other members in the Zhou lab for discussion. This work was supported by the Shriners Hospitals for Children research grants (8610 and 8750 to C.Z.) and by a UC Davis Health System Research Award (to C.Z.).

Open Access This article is distributed under the terms of the Creative Commons Attribution Noncommercial License which permits any noncommercial use, distribution, and reproduction in any medium, provided the original author(s) and source are credited.

\section{References}

Archer CW, Dowthwaite GP, Francis-West P (2003) Development of synovial joints. Birth Defects Res C Embryo Today 69:144-155

Barolo S (2006) Transgenic Wnt/TCF pathway reporters: all you need is Lef? Oncogene 25:7505-7511

Baron R, Rawadi G, Roman-Roman S (2006) Wnt signaling: a key regulator of bone mass. Curr Top Dev Biol 76:103-127

Bernard P, Fleming A, Lacombe A, Harley VR, Vilain E (2008) Wnt4 inhibits beta-catenin/TCF signalling by redirecting beta-catenin to the cell membrane. Biol Cell 100:167-177

Church VL, Francis-West P (2002) Wnt signalling during limb development. Int J Dev Biol 46:927-936

DasGupta R, Fuchs E (1999) Multiple roles for activated LEF/TCF transcription complexes during hair follicle development and differentiation. Development 126:4557-4568

Day TF, Yang Y (2008) Wnt and hedgehog signaling pathways in bone development. J Bone Joint Surg Am 90(Suppl 1):19-24
Fuchs E (2008) Skin stem cells: rising to the surface. J Cell Biol 180:273-284

Gordon MD, Nusse R (2006) Wnt signaling: multiple pathways, multiple receptors, and multiple transcription factors. J Biol Chem 281:22429-22433

Guo X, Day TF, Jiang X, Garrett-Beal L, Topol L, Yang Y (2004) Wnt/beta-catenin signaling is sufficient and necessary for synovial joint formation. Genes Dev 18:2404-2417

Hartmann C (2006) A Wnt canon orchestrating osteoblastogenesis. Trends Cell Biol 16:151-158

Hartmann C, Tabin CJ (2001) Wnt-14 plays a pivotal role in inducing synovial joint formation in the developing appendicular skeleton. Cell 104:341-351

Karsenty G, Wagner EF (2002) Reaching a genetic and molecular understanding of skeletal development. Dev Cell 2:389-406

Koyama E, Shibukawa Y, Nagayama M, Sugito H, Young B, Yuasa T, Okabe T, Ochiai T, Kamiya N, Rountree RB, Kingsley DM, Iwamoto M, Enomoto-Iwamoto M, Pacifici M (2008) A distinct cohort of progenitor cells participates in synovial joint and articular cartilage formation during mouse limb skeletogenesis. Dev Biol 316:62-73

Krishnan V, Bryant HU, Macdougald OA (2006) Regulation of bone mass by Wnt signaling. J Clin Invest 116:1202-1209

Kronenberg HM (2003) Developmental regulation of the growth plate. Nature 423:332-336

Leucht P, Minear S, Ten Berge D, Nusse R, Helms JA (2008) Translating insights from development into regenerative medicine: the function of Wnts in bone biology. Semin Cell Dev Biol 19:434-443

Liu F, Kohlmeier S, Wang CY (2008) Wnt signaling and skeletal development. Cell Signal 20:999-1009

Merino R, Macias D, Ganan Y, Economides AN, Wang X, Wu Q, Stahl N, Sampath KT, Varona P, Hurle JM (1999) Expression and function of Gdf-5 during digit skeletogenesis in the embryonic chick leg bud. Dev Biol 206:33-45

Pitsillides AA, Ashhurst DE (2008) A critical evaluation of specific aspects of joint development. Dev Dyn 237:2284-2294

Reya T, Clevers H (2005) Wnt signalling in stem cells and cancer. Nature 434:843-850

Spater D, Hill TP, Gruber M, Hartmann C (2006a) Role of canonical Wnt-signalling in joint formation. Eur Cell Mater 12:71-80

Spater D, Hill TP, O'Sullivan RJ, Gruber M, Conner DA, Hartmann C (2006b) Wnt9a signaling is required for joint integrity and regulation of Ihh during chondrogenesis. Development 133:3039-3049

Storm EE, Kingsley DM (1999) GDF5 coordinates bone and joint formation during digit development. Dev Biol 209:11-27

Veeman MT, Axelrod JD, Moon RT (2003) A second canon. Functions and mechanisms of beta-catenin-independent Wnt signaling. Dev Cell 5:367-377

Wang YZ, Molotkov A, Song L, Li Y, Pleasure DE, Zhou CJ (2008) Activation of the Wnt/beta-catenin signaling reporter in developing mouse olfactory nerve layer marks a specialized subgroup of olfactory ensheathing cells. Dev Dyn 237:3157-3168

Yang Y (2003) Wnts and wing: Wnt signaling in vertebrate limb development and musculoskeletal morphogenesis. Birth Defects Res C Embryo Today 69:305-317

Zhang Y, Ryan JA, Di Cesare PE, Liu J, Walsh CA, You Z (2007) Doublecortin is expressed in articular chondrocytes. Biochem Biophys Res Commun 363:694-700

Zhou CJ, Wang YZ, Yamagami T, Zhao T, Song L, Wang K (2009) Generation of Lrp6 conditional gene-targeting mouse line for modeling and dissecting multiple birth defects/congenital anomalies. Dev Dyn (Epub ahead of print). doi:10.1002/dvdy.22054 